\title{
Revisión del concepto de inmunidad poblacional, a propósito de la epidemia COVID-19 y del desarrollo de vacunas
}

\author{
Review of the concept of herd immunity, in the context of COVID-19 epidemic and the \\ development of vaccines
}

\author{
Mauricio Canals L. ${ }^{1}$
}

${ }^{1}$ Programa de Salud Ambiental, Escuela de Salud Pública y Departamento de Medicina, Facultad de Medicina, Universidad de Chile.

Sin conflictos de interés.

Recibido: 7 de agosto de 2020 (segunda versión 15 de febrero de 2021) / Aceptado: 3 de julio de 2021

\section{Resumen}

Se revisa brevemente el concepto de inmunidad de grupo, poblacional o efecto "rebaño", mostrando que algunas ideas popularizadas no corresponden al concepto original. Se establece la relación con los números reproductivo básico y efectivo, enfatizándose que el umbral para el efecto rebaño no indica el número de individuos que se contagiarán en una epidemia. Se establece la relación con el umbral de vacunación efectiva y su relación con la efectividad de la vacuna. Se analiza el efecto reductor del umbral de inmunidad de rebaño producido por la heterogeneidad de transmisión y mezcla en la población y la existencia de subpoblaciones aisladas lo que podría llegar a ser importante y que podría explicar los bajos niveles de seroprevalencia post-epidemia de algunos lugares, ayudando a mitigar nuevos brotes.

Palabras clave: inmunidad poblacional; concepto; número reproductivo; vacunación; cálculo.

\section{Introducción}

$\mathrm{L}$ a inmunidad poblacional, comunitaria o de grupo se puede definir como la proporción de inmunidad adquirida por un grupo $^{1}$. Contiene la idea que el riesgo de infección entre individuos susceptibles se reduce en presencia de muchos inmunes, lo que se conoce como "efecto rebaño" o lo que J. Brownlee llamaba la dilución de los susceptibles en los casos ${ }^{2}$. Algunos autores lo usan para describir la proporción de individuos inmunes en una población, otros como la proporción umbral para disminución de la incidencia de

\section{Abstract}

The concept of herd immunity is briefly reviewed, showing that some popularized ideas do not correspond to the original concept. The relationship with the basic and effective reproductive numbers is established. It is pointed out that the threshold for the herd effect does not indicate the number of individuals that will be infected in an epidemic. The relationship with the effective vaccination threshold and its relationship with the effectiveness of the vaccine are established. The reducing effect of the herd immunity threshold produced by the heterogeneity of transmission and mixing in the population and the existence of isolated subpopulations are analyzed, which could be important and could explain the low levels of post-epidemic seroprevalence in some places helping to mitigate new outbreaks.

Keywords: herd immunity; concept; reproductive number; vaccination; calculation. 
importantes para entender el desarrollo y después se establecerán los conceptos y consecuencias más importantes de las definiciones.

\section{Número reproductivo básico $\left(R_{0}\right)$}

Se define número reproductivo básico $\left(R_{0}\right)$ de una enfermedad infecciosa como el número de casos nuevos a partir de un caso bajo el supuesto de un $100 \%$ de susceptibles (supuesto que se usa al inicio de un brote) ${ }^{10}$. Aunque el concepto es sencillo, su estimación no es tan simple. Se puede estimar de varias maneras: La primera es a partir de modelos tipo Susceptible-Infectado-Resistente (SIR) y Susceptible-Latente-Infectado-Resistente (SEIR) por varios métodos, entre ellos el más común es el de la matriz de la siguiente generación ${ }^{11,12}$. Por ejemplo en un modelo SIR básico: $R=\frac{N \beta}{(\gamma+\mu)}$, donde $\beta$ es el coeficiente de transmisión, $\gamma$ la tasa de recuperación y $\mu$ la tasa de mortalidad, $\mathrm{N}$ es la población inicial de susceptibles $\beta$ que puede no incluirse, dependiendo de cómo se parametrice $\beta$. En un SEIR básico: $R=\frac{N v \beta}{(v+\mu)(\gamma+\mu)}$, donde $v$ es la tasa de transferencia de latente a infeccioso ${ }^{10}$ (Figura 1).

\footnotetext{
Métodos basados en modelos

En un SIR básico: $\quad R_{0}=\frac{N \beta}{(\gamma+\mu)}$

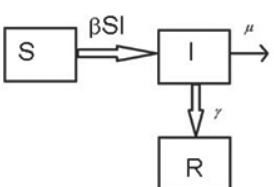

En un SEIR básico: $\quad R_{0}=\frac{N v \beta}{(v+\mu)(\gamma+\mu)}$

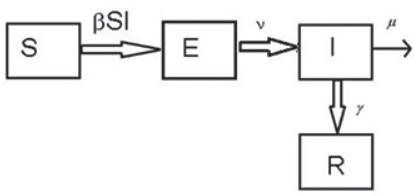

Métodos basados en los datos

Métodos a prior

$$
R_{0}=\frac{I(t+\tau)}{I(t)}=1+r \tau
$$

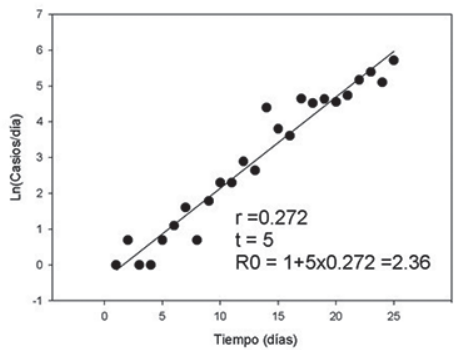

Métodos a posteriori $R_{0}=1+\frac{e 0}{E}, \quad R_{0}=\frac{\ln \left(\frac{1}{s}\right)}{1-s}$

Figura 1. Algunos métodos de cálculo del número reproductivo básico $\left(R_{0}\right)$. En el recuadro un ejemplo basado en la estimación de la pendiente en el gráfico del logaritmo natural de los casos nuevos diarios, considerando un intervalo serial de 5 días. Aquí, $S$ son los susceptibles, $E$ los latentes, I los infectados y $R$ los resistentes (recuperados y fallecidos); $\beta$ es el coeficiente de transmisión, $\gamma$ la tasa de recuperación y $\mu$ la tasa de mortalidad, $v$ es la tasa de transferencia de latente a infeccioso, $N$ es la población inicial de susceptibles, $\tau$ es el intervalo serial, $r$ la tasa de crecimiento exponencial de los casos diarios, $e_{0}$ la esperanza de vida al nacer, $E$ la edad media de infección y la proporción de susceptibles al final de la epidemia.
}

La segunda manera de estimarlo es a partir de los datos con métodos a posteriori (después de ocurrida la epidemia) y con métodos de estimación al inicio de la epidemia basados en el crecimiento cuasi exponencial de ésta ${ }^{10,13}$. Un método simple y rápido es estimar $R_{0}$ con $R_{0}=1+\mathrm{r} \tau$ : Considerando la curva de casos nuevos por día hasta el punto de quiebre de la curva, punto en el que se supone comienzan a intervenir otros factores como las intervenciones epidemiológicas y deja de existir un crecimiento exponencial se puede hacer una recta de regresión entre Ln (Casos nuevos) y t; estimar $r$ a través de la pendiente de esta recta y conociendo $\tau$ estimar $R_{0}{ }^{10}$.

\section{Número reproductivo efectivo $(R(t))$}

El número reproductivo efectivo tiene exactamente la misma definición que $R_{0}$, pero bajo una proporción de susceptibles, variable en el tiempo y frente a medidas de mitigación y control. Como la epidemia se desarrolla en un escenario cambiante de susceptibles y bajo diferentes intervenciones epidemiológicas, $R(t)$ permite seguir la evolución de una epidemia en el tiempo. Si $R_{0}>1$, se tienen infectados en la población. Su estimación se puede hacer por diversos métodos. Entre ellos, los métodos de Wallinga \& Tenuis $2004^{14}$, Wallinga \& Lipsitchs $2006^{15}$, Bettencourt \& Ribeiro $2008^{16}, \mathrm{RKI}^{17}$ y Cori y cols. $(2013)^{18}$. En una revisión reciente (19) recomiendan la aproximación Bayesiana de Cori y cols., para la estimación en tiempo real de R(t), lo que actualmente se ha realizado en Chile en esta epidemia ${ }^{20}$.

\section{Inmunidad poblacional}

Klaus Dietz en $1975^{21}$, estudiando las condiciones para una endemia en un modelo SIR edad-estructurado, derivó tres relaciones importantes: La primera es que la condición de existencia infectados en la población está dada por: $R_{0}=\left(1+\frac{e_{0}}{A}\right)$, donde $e_{0}$ es la esperanza de vida

$$
\begin{aligned}
& \text { RKI } \quad R_{t, 7}=\frac{\bar{E}_{t}^{7}}{\bar{E}_{t-\tau}^{7}}=\frac{\sum_{s=t-6}^{t} E_{s}}{\sum_{s=t-6}^{t} E_{s-\tau}} . \\
& \text { Cori et al (2013). } \quad R(t)=\frac{I(t)}{\int I(t-T) w(T) d T}
\end{aligned}
$$

Figura 2. Fórmulas de estimación del número reproductivo efectivo con los métodos RKI $\left(R_{t}\right)^{17}$ y de Cori ${ }^{18}$. En RKI, $E_{t}$ representa el número de casos en el día t y la barra con el número 7 sobre la letra significa promedio móvil de orden 7. En la fórmula de Cori el número reproductivo efectivo $R(t)$ se expresa en función de la incidencia en el tiempo " $\mathrm{t}$ " $(I(t))$ y la suma (integral) de las incidencias previas $(I(t-T))$ por la densidad de probabilidades de transmisión $w(T)$. 
inmunidad, el alcanzar la inmunidad de rebaño en forma natural es cuestión de tiempo. La pérdida de inmunidad no afectará el valor del umbral p*28; sin embargo, si existe pérdida de la inmunidad, el que se logre alcanzar este umbral va a depender de la relación entre la tasa de reclutamiento de infectados y la tasa de pérdida de la inmunidad. Mientras mayor sea la tasa de pérdida de inmunidad, más tarde se alcanzará el umbral p*, y aunque este se alcance existirán nuevos brotes (recurrencia epidémica) por el elevado reclutamiento de susceptibles ${ }^{28}$. fácil de entender si consideramos que en el equilibrio cada uno de estos $1 / R_{0}$ susceptibles debiera producir un solo nuevo caso, es decir el número reproductivo efectivo es $R(t)=1 / R_{0} \times R_{0}=1$.

La tercera, tal vez la más importante y relacionada con la anterior, es que durante el transcurso de una epidemia la proporción de susceptibles es menor que 1 y entonces sólo los $q$ susceptibles serán capaces de dejar nuevos casos, mientras $p=1-q$ no lo harán. Es decir, se producirán $R(t)=q R_{0}$ casos nuevos, lo que establece la relación entre $R_{0}$ y el número reproductivo efectivo, resultado que ya CEG Smith había propuesto en $1970^{24}$.

De este resultado se puede proponer que la condición necesaria para que disminuya la incidencia de casos es $R(t)<1$. Es decir $q R_{0}<1$ lo que es equivalente a $(1-p) R_{0}$ $<1$ y también equivalente a: $p>\left(1-1 / R_{\theta}\right)$. Entonces, el

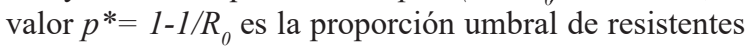
(contagiados o vacunados) que hace que $\mathrm{R}(\mathrm{t})<1 \mathrm{y}$, en consecuencia, comience a disminuir la incidencia de contagios $^{1,2,5,21,24}$. Así, bajo el supuesto de población homogénea, $p^{*}$ corresponde al valor umbral de inmunidad poblacional o de grupo (o para alcanzar el efecto "rebaño"). Se usa entonces como umbral de población que es necesario vacunar con una vacuna $100 \%$ efectiva para que, de instalarse un brote, éste sea pasajero y autolimitado ${ }^{5,25}$.

Observamos que este umbral no evita que existan algunos casos, como por ejemplo el pequeño brote de sarampión que ocurrió en Chile en 2018-201923 y tampoco significa que sólo una proporción $p^{*}$ se va a infectar en una epidemia. Corresponde al umbral a partir de cual $R(t)$ se hace menor que 1 y la incidencia diaria desciende. La proporción de individuos que se infectan en una epidemia $(p \infty=1-q \infty)$ dejada a su evolución natural en una población con mezcla homogénea está dada por la relación: $R_{0}=\frac{\ln \left(\frac{1}{q \infty}\right)}{1-q \infty}$, relación que se obtiene de sumar e integrar las ecuaciones diferenciales para susceptibles en infectados en un modelo $\mathrm{SIR}^{21,26,27}$. Así, por ejemplo, para un $R_{0}=$ 2,35 , la inmunidad de rebaño se alcanzaría con $57,4 \%$ de infectados, pero dejada la epidemia a su evolución natural, llegaría a contagiar a $87 \%$ de la población.

Otro aspecto importante a considerar es la posibilidad de alcanzar la inmunidad de rebaño. Uno de los supuestos de los modelos tipo SIR y SEIR es que no existe pérdida de la inmunidad una vez que se adquirió la infección. Sin embargo, esto no siempre es así. Cuando no hay pérdida de

\section{Vacunas e inmunidad poblacional}

Hay dos aspectos importantes a destacar de los resultados anteriores. El primero es que todos los modelos epidemiológicos tipo SIR o SEIR se basan en el supuesto de una mezcla homogénea de la población, lo que en general nunca se cumple, y el segundo es que si se va a considerar como umbral de vacunación $(V c)$, se debería considerar la efectividad $(E)$ de la vacuna ${ }^{1}$. Esta se define como: reducción en la transmisión de la infección hacia y desde los individuos vacunados en comparación con los individuos control en la misma población, lo que es un concepto análogo a la eficacia de la vacuna convencional, pero midiendo la protección contra la transmisión en lugar de la protección contra la enfermedad. En este caso: $V c=\frac{\left(1-\frac{1}{R_{0}}\right)}{E}$. Importante a considerar en las vacunas es la tasa de pérdida de la inmunidad inducida por la vacuna. Si la inmunidad no es permanente y existe una tasa de pérdida de inmunidad " $\phi$ ”, entonces, se ha propuesto que en $V c$ hay que considerar un factor de corrección: $V C=\frac{\left(1-\frac{1}{R_{0}}\right)}{E\left(\frac{1 / e_{0}}{1 / e_{0}+\phi}\right)}$, donde $e_{0}$ es la esperanza de vida al nacer ${ }^{29}$. Es decir, el control del COVID-19 mediante vacunas depende del $R_{0}$, de la efectividad de la vacuna y de la permanencia de la inmunidad inducida por la vacuna.

El efecto de la heterogeneidad de la población es más complejo. Algunos modelos han considerado interacción entre grupos de edad $^{30} \mathrm{y}$ otros modelos espacialmente explícitos han parametrizado usando los datos de transporte ${ }^{31}$. Recientemente se ha reportado que cuando existe mezcla heterogénea de la población entre individuos de diferentes edades, cada uno con diferentes coeficientes de transmisión, en el caso de un $R_{0}=2,5$, el umbral p* (y por tanto $V c$ ) puede descender de 60 a $55,8 \%$. Si existe social también con diferentes coeficientes de transmisión, el umbral p* puede descender de 60 a 46,3\% y cuando se cumplen ambas condiciones puede descender de 60 a $43 \%{ }^{32}$. En otro estudio se ha considerado una población heterogénea con un subgrupo poblacional con resistencia a la infección con diferentes niveles de mezcla entre las subpoblaciones. En éste se ha encontrado que el umbral mezcla heterogénea entre grupos de diferente actividad 
Tabla 1. Relación entre el número reproductivo básico $\left(R_{0}\right)$ la proporción umbral para inmunidad poblacional $\left(p^{*}\right)$ y proporción de infectados al final de una epidemia dejada a su evolución natural, con población homogénea $(p \infty=1-s)$

\begin{tabular}{|ccc}
\hline $\boldsymbol{R}_{\boldsymbol{o}}$ & $\boldsymbol{p}^{*}(\%)$ & $\boldsymbol{p}(\%)$ \\
1,03 & 2,52 & 5 \\
1,08 & 7,70 & 15 \\
1,15 & 13,10 & 25 \\
1,23 & 18,75 & 35 \\
1,33 & 24,73 & 45 \\
1,36 & 26,60 & 48 \\
1,45 & 31,12 & 55 \\
1,62 & 38,08 & 65 \\
1,66 & 39,57 & 67 \\
1,91 & 47,61 & 77 \\
2,35 & 57,36 & 87 \\
2,56 & 60,91 & 90 \\
\hline
\end{tabular}

p* desciende en la medida que aumenta la proporción de resistentes y puede descender a valores tan bajos como 10,20 o $30 \%$ cuando existe una mayor mezcla entre las sub-poblaciones, es decir, cuando los resistentes se mezclan con la sub-población no-resistente ${ }^{33}$. Esto se explicaría porque los resistentes formarían agrupaciones que evitarían la propagación espacial del virus y producirían un efecto de "dilución" de los casos (en el sentido de Brownlee) reduciendo la probabilidad de transmisión. Estos autores proponen que, para la actual epidemia, las medidas de seropositividad del $10-20 \%$ son totalmente compatibles con los niveles locales de inmunidad que se han acercado a $\mathrm{p}^{*}$; pensando que el riesgo y la escala de resurgimiento podrían ser más bajos de lo que se percibe actualmente.

Aunque los valores obtenidos por estos autores son discutibles y atribuibles al modelo y los parámetros usa- dos, todos los modelos y la lógica sugieren que ante una heterogeneidad de la población con diferentes coeficientes de transmisión o aislamiento entre sub-poblaciones, la probabilidad de contagio será menor, y en consecuencia, el umbral necesario para que se alcance una adecuada inmunidad poblacional será también menor, lo que por una parte podría estar avalado por los niveles de seroprevalencia reportados después de los brotes en varias ciudades ${ }^{34-38} \mathrm{y}$, por otra parte, sugerirían que en algunas localidades podría existir suficiente inmunidad de rebaño para mitigar sustancialmente nuevos brotes ${ }^{33}$.

\section{Conclusiones}

El concepto de inmunidad poblacional o de grupo corresponde a la inmunidad alcanzada por la población ante la propagación de una enfermedad infecciosa o campaña de vacunación. El umbral necesario para alcanzar un nivel de inmunidad que condicione un descenso en la incidencia de una epidemia o haga insostenible y autolimitado un brote se conoce como el umbral ( $\left.\mathrm{p}^{*}\right)$ para "efecto rebaño" y se encuentra determinado por la relación $R(t)=q R_{0}<1$.

El umbral para el efecto rebaño no indica el número de individuos que se contagiarán en una epidemia.

La proporción de individuos de una población homogénea que se debieran vacunar para evitar brotes sostenidos $(V c)$ depende fundamentalmente del umbral $p^{*} \mathrm{y}$ de la efectividad de la vacuna $(E)$.

La heterogeneidad de transmisión y mezcla en la población y la existencia de subpoblaciones aisladas producen una disminución en el umbral $p^{*}$ que puede llegar a ser importante y que podría explicar los bajos niveles de seroprevalencia post-epidemia de algunos lugares, lo que podría ayudar a mitigar nuevos brotes.

Agradecimientos. Auspiciado por el proyecto ANIDCOVID 0960

\section{Referencias bibliográficas}

1.- Fine P, Eames K, Heymann D L. "Herd Immunity": a rough guide. Clin Infect Dis. 2011; 52 (7): 911-6. doi: 10.1093/cid/cir007.

2.- Fine P E M. John Brownlee and the measurement of infectiousness: an historical study in epidemic theory. J. R. Statist. Soc A 1979; 142 (3): 347-62. https://doi. org/10.2307/2982487.

3.- Fox J P, Elveback L, Scott W, Gatewood L, Ackerman E. Herd immunity: basic concept and relevance to public health immunization practices. Am J Epidemiol. 1971; 94: 179-89. doi: 10.1093/oxfordjournals.aje.a121310.
4.- Anderson R M, May R M. Vaccination and herd immunity to infectious diseases. Nature 1985; 318: 323-9. https://doi. org/10.1038/318323a0.

5.- Fine P E M. Herd immunity: history, theory, practice. Epidemiol Rev. 1993; 15: 265-302. doi: 10.1093/oxfordjournals.epirev.a036121.

6.- Fine P E M, Mulholland K, Scott J A, Edmunds WJ. Chapter 77. Community Protection. In: Plotkin's Vaccines. Plotkin SA, Orenstein WA, Offit PA, Edwards KM, eds. Vaccines. 7th ed. Chapter 71. Philadelphia, PA: Elsevier Inc., 2018: 1512-31.

7.- John T J, Samuel R. Herd immunity and herd effect: new insights and definitions.
Eur J Epidemiol. 2000; 16: 601-6. doi: 10.1023/a:1007626510002.

8.- Stephens D S. Vaccines for the unvaccinated: protecting the herd. J Infect Dis. 2008; 197 : 643-5. doi: 10.1086/527402.

9.- Heymann D, Aylward B. Mass vaccination in public health. In: Heymann D, ed. Control of Communicable Diseases Manual. 19th ed. Washington, DC: American Public Health Association, 2008.

10.- Anderson R M. Epidemiology. In Modern Parasitology (Cox FEG ed.). Oxford: Blacwell Scientific Publications; 1993. 75-117pp.

11.- Diekmann O, Heesterbeek J A P, Metz J A J. On the definition and computation of the basic 
reproduction ratio $\mathrm{R} 0$ in models for infectious diseases in heterogeneous populations. J. Math. Biol. 1990; 28: 365-82. https://doi.org/10.1007/ BF00178324.

12.- Diekmann O, Heesterbeek J A P, Roberts M G. The construction of next-generation matrices for compartmental epidemic models. J. R. Soc. Interface. 2010; 7: 873-85. doi: 10.1098/ rsif.2009.0386.

13.- Heffernan J M, Smith R J, Wahl L M. Perspectives on the basic reproductive ratio. J. R. Soc. Interface. 2005; 2: 281-93. doi: 10.1098/rsif.2005.0042, 1-13.

14.- Wallinga J, Teunis P. Different epidemic curves for severe acute respiratory syndrome reveal similar impacts of control measures. Am J Epidemiol. 2004; 160 (6): 509-16. doi:10.1093/ aje/kwh255.

15.- Wallinga J, Lipsitch M. How generation intervals shape the relationship between growth rates and reproductive numbers. Proc Biol Soc. 2007; 274: (1609): 599-604. doi: 10.1098/ rspb.2006.3754.

16.- Bettencourt L M A, Ribeiro R M. Real time Bayesian estimation of the epidemic potential of emerging infectious diseases. PLoS ONE. 2008; May 14; 3 (5): e2185. doi:10.1371/ journal.pone.0002185.

17.- an der Heiden M, Hamouda O. Schätzung der aktuellen Entwicklung der SARS-CoV2-Epidemie in Deutschland - Nowcasting. Epid Bull 2020; 17: 10-6. https://doi. org/10.25646/6692.4.

18.- Cori A, Ferguson N M, Fraser C, Cauchemez $\mathrm{S}$. A new framework and software to estimate time-varying reproduction numbers during epidemics. Am J Epidemiol. 2013; 178 (9): 1505-12. https://doi.org/10.1093/aje/kwt133.

19.- Gostic K M, McGough L, Baskerville E, Abbott S, Joshi K, Tedijanto C, et al. Practical considerations for measuring the effective reproductive number, Rt. doi: https://doi. org/10.1101/2020.06.18.20134858.this version posted June 21, 2020. https://www. medrxiv.org/content/10.1101/2020.06.18.2013 $4858 \mathrm{v} 3$.
20.- Canals M, Cuadrado C, Canals A, Johannessen K, Lefio L A, Bertoglia M P, et al. Epidemic trends, public health response and health system capacity: The Chilean experience in COVID-19 epidemic. Pan Am J Public Health. 2020; 44: https://doi.org/10.26633/RPSP.2020.99.

21.- Dietz K. Transmission and control of arbovirus diseases. In: Ludwig D, Cooke KL editors. Epidemiology. SIAM, Philadelphia, 1975; 10421.

22.- Canals M, Martínez L, Firinguetti L. Dinámica ecológica de enfermedades infecciosas. II parte: Recurrencia epidémica. Rev Med Chile. 1989; 117: 95-100. http://dx.doi.org/10.4067/S003498871999000900008.

23.- Canals M, Avendaño L F, Rojas C. Estimando el número reproductivo efectivo del brote de sarampión 2018-2019 en Chile. Rev Chilena Infectol 2020; 37 (3): 231-6. http://dx.doi. org/10.4067/s0716-10182020000300231.

24.- Smith C E G. Prospects for the control of infectious disease. Proc Roy Soc Med. 1970; 63: 1181-90. PMID: 5530322

25.- Anderson R M, May R M. Infectious diseases of humans: dynamics and control. Oxford, UK: Oxford University Press, 1991.

26.- Bailey N. 1975. The mathematical theory of infectious diseases. Griffin, London.

27.- Bailey N. 1975. The mathematical theory of infectious diseases and its applications. Griffin, London.

28.- Bj申rnstad B J, Shea K, Krzywinski M, Altman N. The SEIRS models for infectious diseases dynamics. Nat Methods 2020, 17: 555-8. doi: 10.1038/s41592-020-0856-2.

29.- Scherer A, McLean A. Mathematical models of vaccination. Br Med Bull 2002; 62: 187-99. doi: $10.1093 / \mathrm{bmb} / 62.1 .187$.

30.- Mossong J, Hens N, Jit M, Beutels P, Auranen $\mathrm{K}$, et al. Social contacts and mixing patterns relevant to the spread of infectious diseases. PLoS Med. 2008; Mar 25; 5 (3): e74. doi:10.1371/journal.pmed.0050074.

31.- Colizza V, Barrat A, Barthe 'lemy M, Vespigniani A. The role of the airline transportation network in the prediction and predictability of global epidemics. Proc Natl Acad Sci USA 2006; 2006103 (7) 2015-2020. https://doi.org/10.1073/pnas.0510525103.

32.- Britton T, Ball F, Trapman P. A mathematical model reveals the influence of population heterogeneity on herd immunity to SARSCoV-2. Science 2020; Aug 14; 369 (6505): 846-9. doi: 10.1126/science.abc6810.

33.- Lourenço J, Pinotti F, Thompson C, Gupta S. The impact of host resistance on cumulative mortality and the threshold of herd immunity for SARS-CoV-2. 2020; https://doi.org/10.1101 /2020.07.15.20154294doi: medRxiv pr.

34.- Stadlbauer D, Tan J, Jiang K, Hernández M, Fabre S, Amanat F, et al. Seroconversion of a city: longitudinal monitoring of SARS-CoV-2 seroprevalence in New York City, MedRxiv preprint. doi: 10.1101/2020.06.28.20142190.

35.- Pollán M, Pérez-Gómez B, Pastor-Barriuso R, Oteo J, Hernán M A, Pérez-Olmeda $\mathrm{M}$, et al. ENE-COVID Study Group, Prevalence of SARS-CoV-2 in Spain (ENECOVID): a nationwide, population-based seroepidemiological study. Lancet 2020; 306: 535-44. doi: 10.1016/S0140-6736(20)31483-5.

36.- Stringhini S, Wisniak A, Piumatti G, Azman A S, Lauer S A, Baysson, et al. Seroprevalence of anti-SARS-CoV-2 IgG antibodies in Geneva, Switzerland (SEROCoV-POP): a populationbased study. Lancet 2020; 396 (10247): 313-19. doi: 10.1016/S0140-6736(20)31304-0.

37.- Thompson C, Grayson N, Paton R, Lourenço J, PenmanB, Lee L N, et al. Neutralising antibodies to SARS coronavirus 2 in Scottish blood donors - a pilot study of the value of serology to determine population exposure. medRxiv. BMJ Yale. doi: 10.1101/2020.04.13.20060467.

38.- Percivalle E, Cambiè G, Cassaniti I, Nepita E V, Maserati R, Ferrari A, et al. Prevalence of SARS-CoV-2 specific neutralising antibodies in blood donors from the Lodi Red Zone in Lombardy, Italy, as at 06 April 2020. Euro Surveill. 2020; 25 (24): 2001031. doi: 10.2807/1560-7917. ES.2020.25.24.2001031. 